\title{
Influence of cerebellar stereotactic stimulation on left-right electrodermal information transference in a patient with cerebral palsy
}

This article was published in the following Dove Press journal:

Neuropsychiatric Disease and Treatment

28 November 2013

Number of times this article has been viewed

Petr Bob ${ }^{1,2}$

Tomas Galanda ${ }^{3}$

Peter Jombik ${ }^{4}$

Jiri Raboch'

Miroslav Galanda ${ }^{3}$

'Center for Neuropsychiatric

Research of Traumatic Stress,

Department of Psychiatry, Ist Faculty

of Medicine, Charles University,

Prague, Czech Republic; ${ }^{2}$ Central

European Institute of Technology,

Faculty of Medicine, Masaryk

University, Brno, Czech Republic;

${ }^{3}$ Department of Neurosurgery,

Slovak Medical University,

Roosevelt Hospital, Banska Bystrica,

Slovak Republic; ${ }^{4}$ Department of

Neurology, Laboratory of Clinical

Neurophysiology, Zvolen Hospital,

Zvolen, Slovak Republic
Correspondence: Petr Bob,

Department of Psychiatry,

Charles University, Ist Faculty

of Medicine, Ke Karlovu II,

12800 Prague, Czech Republic

Tel +42022 4965314

Email petrbob@netscape.net
Background and objectives: Recent evidence indicates that cerebral palsy is connected to specific autonomic dysregulation between sympathetic and parasympathetic efferent pathways, likely linked to hemispheric influences. These findings suggest a hypothesis that contralateral interhemispheric disinhibition, which may occur on various levels of brain processing including motor functions, could be linked to specific functional dysregulation and structural lesions, which may play a specific role in the modulation of autonomic functions and lead to autonomic dysregulation in cerebral palsy.

Method: With the aim of comparing autonomic functions as they relate to interhemispheric modulatory influences during therapeutically indicated stereotactic cerebellar stimulation, we have performed bilateral electrodermal activity measurement and calculations of pointwise transinformation (PTI) in a patient with cerebral palsy. Measurement was performed during therapeutic deep cerebellar stimulation in two cerebellar areas in anterior cerebellar lobe-culmen (left electrode) and central lobule-superior cerebellar peduncle (right electrode).

Results: The results indicate that information transference (PTI) is able to distinguish the states related to specific cerebellar stimulations and that lowest levels of the PTI have been found during stimulation of the central lobule-superior cerebellar peduncle (electrode deepest contact 1), indicating a significantly increased level of inhibition between the left and right sides.

Conclusion: The results may present potentially useful clinical findings indicating that increased PTI calculated from electrodermal activity could indirectly indicate disinhibitory activity as a possible indicator of a failure of interhemispheric communication that could explain some specific pathogenetic mechanisms in cerebral palsy. Nevertheless, these results need detailed confirmation in further research, as well as reliable clinical evaluation of their usefulness in the therapy of cerebral palsy.

Keywords: autonomic laterality, bilateral electrodermal activity, cerebral palsy, epileptiform activity, interhemispheric interaction, stereotactic cerebellar stimulation

\section{Introduction}

According to several recent findings, cerebral palsy is linked to specific autonomic dysregulation, likely caused by sympathovagal imbalance. ${ }^{1,2}$ The dysregulation between sympathetic and parasympathetic efferent pathways is likely linked to hemispheric influences related to autonomic modulation that results from the cerebral lesions that typically occur in cerebral palsy patients. ${ }^{1,3,4}$ Several clinical findings also indicate that a significant number of patients with cerebral palsy respond well to vagal nerve stimulation, and that treatment of interictal epileptiform discharges in cerebral palsy patients who have not clinical epilepsy may well improve clinical prognosis. ${ }^{5,6}$ 
On the basis of similar principles influencing inhibitory systems and autonomic dysregulation, there is also clinical evidence that several methods of cerebellar stimulation may significantly improve progress of the disease. ${ }^{7-12}$ For example, Riklan et a ${ }^{13}$ reported tension reduction and improved alertness after chronic cerebellar stimulation for the relief of spasticity in cerebral palsy or intractable epileptic seizures, and they also reported amelioration of tension and anxiety. The epileptic patients primarily reported increased alertness. Although the mechanisms of these changes are not clearly understood, these various clinical and experimental data strongly suggest that cerebral palsy and epilepsy may share some basic pathogenetic mechanisms that are responsible for very strong occurrence of epilepsy in cerebral palsy patients. $., 7,14$

In this context, data from animal models suggest a hypothesis of contralateral interhemispheric disinhibition that may occur on various levels of brain processing, including motor functions, and which could be caused by specific functional dysregulation and structural lesions. ${ }^{15}$ These dysfunctions may play a specific role in the modulation of autonomic functions and lead to autonomic dysbalance on the basis of a failure of interhemispheric communication. This in turn could explain some specific pathogenetic mechanisms connecting cerebral palsy and epilepsy.

With the aim of assessing the specific mechanisms of interhemispheric modulatory systems on integrity and balance of the autonomic nervous system, we have measured bilateral electrodermal activity (EDA). EDA specifically enables us to assess ipsilateral modulation from the left hemisphere on left autonomic activation and the right hemisphere on right autonomic activation. In this context, recent evidence shows that EDA is governed by ipsilateral limbic modulation influences and correlates with amygdala activity, and that other bilateral structures, such as the ventromedial and dorsolateral prefrontal cortices, anterior cingulate gyrus, parietal lobe, insula, hippocampus, and cerebellum, are involved in EDA modulation. ${ }^{16-18}$ Current evidence also shows that EDA reflects a sympathetic axis of the autonomic nervous system and innervations of dermal sweat glands, and provides an index of emotional arousal and stress. ${ }^{16,19}$ On the basis of these mechanisms influencing EDA, we have used EDA records for analysis, reflecting nonlinear interactions between left-right modulatory subsystems regulating EDA. We have calculated the left-right information transfer that likely reflects interhemispheric information flow and sensitive changes in laterality related to modulatory influences on the sympathetic response.

\section{Methods}

\section{Patient}

A 13-year-old boy suffering from cerebral palsy developed spastic quadriparesis with a pronounced startle reaction that seriously disabled his voluntary activity (patient in wheelchair). The patient had two deep brain stimulation electrodes (Medtronic model 3389; Minneapolis, MN, USA) stereotactically implanted (September 6, 2011) in the anterior lobe of the cerebellum (culmen and central lobule-superior cerebellar peduncle) from lateral suboccipital regions (Figures 1 and 2). The electrodes were temporarily connected to a transcutaneous extension to allow further clinical tests and neurophysiological examination, and effects of the stimulation were assessed and verified.

After verification of the effectiveness of stimulation, an internal pulse generator (Medtronic Activa PC) was implanted (September 12, 2011) and stimulation programs were set for therapeutic stimulation (frequency, $250 \mathrm{~Hz}$; pulse width, 0.180 milliseconds; amplitude: right electrode, $1.1 \mathrm{~V}$, and left electrode, $2.7 \mathrm{~V}$; and cycling mode of stimulation, on for 15 minutes, off for 3 hours). Using these parameters of stimulation, the patient was relaxed and spasticity decreased. During a test at a slightly higher amplitude (plus 0.2-0.4 V), the patient experienced an immediate feeling of pleasure. However, an even higher stimulation voltage (3-4 V) evoked bilateral aggravation of the pathological posture of the patient and was accompanied with an intense feeling of fear. For therapeutic stimulation, the amplitude was adjusted to the level at which relaxation and decreased spasticity were achieved.

During experimental EDA measurement, the application of monopolar stimulation was performed in the interval from 1 to $1.5 \mathrm{~V}$ with an increase of $0.1 \mathrm{~V}$ in each step. The stimulation lasted 120 seconds. Because of stimulation spasticity and pathological startle, reactions were remarkably reduced and there was improved fluency in speech, mood,

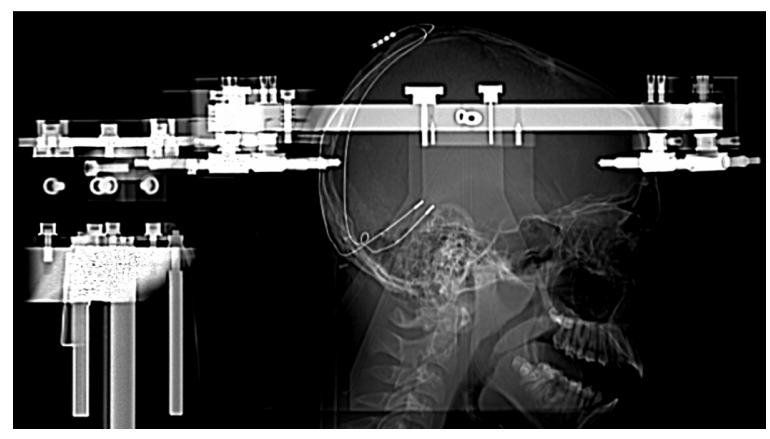

Figure I Lateral skull X-ray. Two deep brain stimulation electrodes (Medtronic model 3389; Minneapolis, MN, USA) were stereotactically implanted bilaterally from retromastoidal burr holes into the anterior lobe of the cerebellum. 


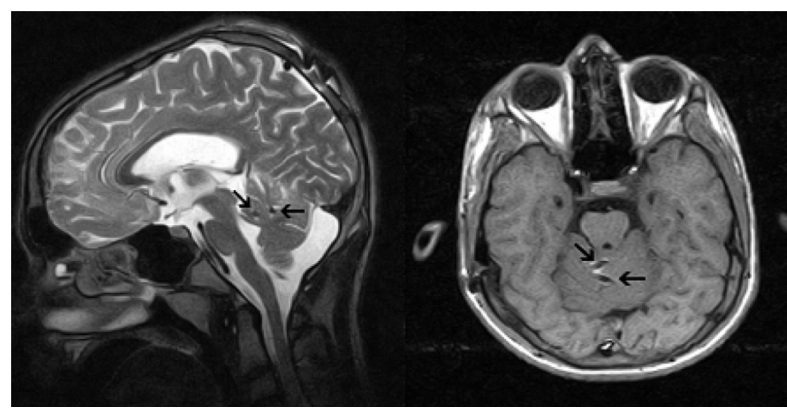

Figure 2 Postoperative axial TI and sagittal T2 weighted magnetic resonance images show active contacts (arrows) of electrodes in the lobulus centralis and in the culmen of the anterior lobe of cerebellum.

and cooperation of the patient during physical therapy. The study was approved by the Slovak Medical University ethical committee (Banska Bystrica, Slovakia), and all participants gave written informed consent.

\section{EDA measurement}

EDA was recorded bilaterally using a two-channel SAM unit and Psylab software (Contact Precision Instruments, London, UK, USA) connected to a personal computer with a sampling frequency of $1,000 \mathrm{~Hz}$. The measurements were performed in a quiet room with a room temperature of about $23^{\circ} \mathrm{C}$. During the experiment, the participant lay on a hospital bed. The measurement was performed using two pairs of $\mathrm{Ag} / \mathrm{AgCl}$ electrodes (8-mm diameter active area) filled with electroconductive paste that were attached to the medial phalanges of the index and middle finger of each hand. EDA measurements lasting 120 seconds were performed during all periods of the cerebellar stimulation, from which (at about 100 seconds) long periods were taken for analysis.

\section{Data analysis}

A practical approach to studying complex dynamical systems is time-series analysis. A postulate of this method is that every dynamic system (eg, the human brain and its functions) is governed and determined by a number of independent variables. However, any real measurement performed on the system cannot provide information about all the variables because a system of high complexity such as the human brain is multidimensional. A time-series analysis therefore represents a mathematical approximation that enables the reconstruction of certain variables underlying the multidimensional dynamics from data obtained from the system during the time. ${ }^{20}$ The data may provide, for example, a psychophysiological measurement performed on the system during an experiment. Because observational data reflect only a few real independent variables of a system, approximation of the dynamic system behavior therefore uses a finite number of (mathematically reconstructed) variables to approximate states of the system or relationships between the subsystems. In this context, it is possible to use a measure of the mutual interaction and information flow between subsystems that may be computed in the phase space, such as pointwise transinformation (PTI). This coupling measure also takes into account nonlinear dependencies and can be applied to nonstationary time-series. The PTI of two observable quantities has been derived from Shannon's information concept and is calculated from the probability densities of the observables in the phase space. ${ }^{21,22}$ In practice, this is done by calculation of empirical point densities in the neighborhood of the reconstructed trajectory. Therefore, the values of the PTI depend on the selected search radius $r$, the range of PTI values is not normalized, and negative PTI values may occur in experimental data. However, the increase or decrease of the PTI level over time still provides information about changes in the coupling behavior of the investigated systems. The time resolution of the PTI is principally limited merely by the sampling rate, and enables us to study very fast changes in coupling behavior. ${ }^{21}$

The defining formula for the $i$-th time step is:

$$
Y=I(\delta, r, i)=\log _{2} \frac{P_{i}^{x 1 \times 2}(\delta, r)}{P_{i}^{x 1}(r) P_{i}^{x 2}(r)}
$$

where the transinformation $I$ is a function of the relative shift and relative phase space radius $r$, given for every sample point $i$. It denotes the probability of finding a point of the reconstructed trajectory within a sphere of radius $r$ around the $i$-th phase space point and refers to the phase spaces of the time series $x 1$ and $x 2$.

In principle, PTI reflects information transfer in a resting state as well as during experimental periods, which in this study were specifically linked to cerebellar stimulation. Statistical evaluation of PTI values (in bits) reflecting both information transfers between the left and right sides and laterality indices was performed, using software package Statistica version 8.0 (StatSoft, Tulsa, OK, USA), and included descriptive statistics, $t$ test, and nonparametric test for dependent samples.

\section{Results}

The results show that during stimulation, EDA progressively increased as a result of stimulation and shows, in a certain sense, specific "phase transitions" related to starting stimulation in specific cerebellar structures (Figure 3). 


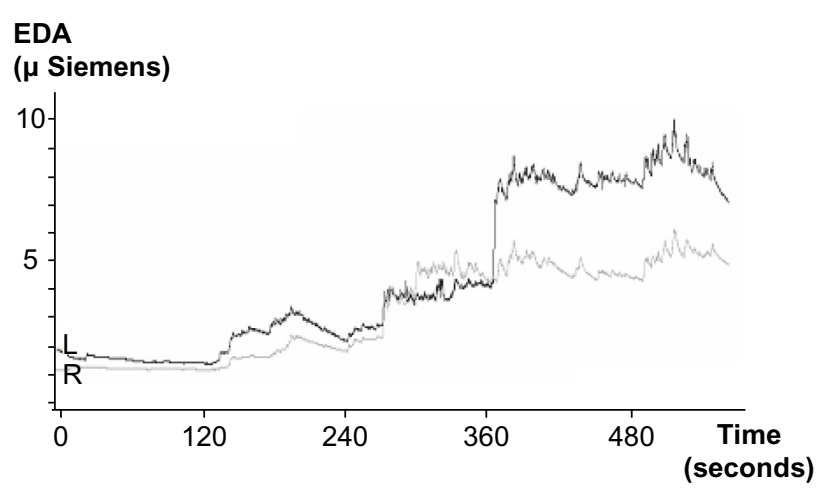

Figure 3 Electrodermal activity (EDA) values during periods of monopolar stimulation of the cerebellar structures. Time from 0 to 120 seconds represents resting state before the stimulation; EDA during the time from 120-240 and from 240-360 seconds reflects increasing stimulation from $\mathrm{I}-\mathrm{I} .5 \mathrm{~V}$ in the left $(\mathrm{L})$ electrode contacts $\mathrm{I}$ and 3 of the anterior lobe-culmen; EDA during the time from 360 to 480 seconds and from 480 to the end reflects increasing stimulation from $\mathrm{I}-\mathrm{I} .5 \mathrm{~V}$ in the right $(\mathrm{R})$ electrode contacts I and 3 of the central lobule-superior cerebellar peduncle.

These stimulation periods were also related to specific changes in left-right electrodermal information transference (PTI), reflecting interhemispheric modulatory influences. The results indicate that the PTI is able to distinguish between these states related to specific cerebellar stimulation, and that the lowest levels of the PTI have been found during stimulation of the central lobule-superior cerebellar peduncle (right electrode, deepest contact 1), indicating a significantly increased level of inhibition between the left and right sides. The result suggests that increased EDA transinformation (PTI) could indirectly indicate increased neural synchrony and disinhibitory activity as a possible indicator of a failure of interhemispheric communication.

The statistical analysis also indicates that the values of PTI (in bits) statistically significantly distinguish all periods during measurement, based on calculation of left-right pairs (Table 1). Statistical comparison using a nonparametric test for multiple comparisons of dependent samples (Friedman's analysis of variance) of averaged values of PTI shows that differences between periods of measurement are statistically significant, at $P<0.0001$ (Table 1). This overall statistical analysis also shows that the lowest level of PTI occurs during stimulation of the central lobule-superior cerebellar peduncle (right electrode contact 1 , using voltage $1.2 \mathrm{~V}$ ), indicating a significantly increased level of inhibition between left and right side.

\section{Discussion}

Results of this study suggest that left-right information transfer calculated from bilateral electrodermal measurement may reflect influences of the brain interhemispheric modulatory systems on the integrity and balance of the autonomic nervous system. In this context, results of this study are in agreement with data from animal models suggesting a hypothesis of contralateral interhemispheric disinhibition, ${ }^{15}$ which may play a specific role in the modulation of autonomic functions and lead to autonomic dysbalance, based on a failure of interhemispheric communication. Consequences of this contralateral interhemispheric disinhibition may occur at various levels of brain processing, including motor functions, and might be caused by specific functional dysregulations and structural lesions that could explain some specific pathogenetic mechanisms connecting cerebral palsy with epilepsy.

In this context, results of this study suggest that specific cerebellar stimulation may influence highly decreased information transference measured by PTI between left and right autonomic neural activities, reflecting brain left and right ipsilateral modulatory influences, and may specifically influence spastic symptoms in cerebral palsy, leading to decreased spasticity and relaxed state. In contrast, highly increased information transference is likely linked to interhemispheric disinhibition, which also is typical for epilepsy and various epileptiform conditions in which epileptiform or epileptic processes tend to extend to the other hemisphere. This spread of epileptic or epileptiform activity significantly influences interhemispheric information transfer that may reflect temporal lobe "epileptogenicity". ${ }^{23,24}$

These findings are also in agreement with data indicating that PTI between left and right autonomic neural

Table I Mean values of EDA and PTI for each experimental period during the cerebellar stimulation

\begin{tabular}{|c|c|c|c|c|c|c|c|c|c|c|c|c|}
\hline \multirow[t]{3}{*}{ Volts } & \multicolumn{6}{|c|}{ Culmen: left electrode } & \multicolumn{6}{|c|}{ Superior cerebellar peduncle: right electrode } \\
\hline & \multicolumn{3}{|c|}{ Contact I } & \multicolumn{3}{|c|}{ Contact 3} & \multicolumn{3}{|c|}{ Contact I } & \multicolumn{3}{|c|}{ Contact 3} \\
\hline & EDA L & EDA R & PTI & EDA L & EDA R & PTI & EDA L & EDA R & PTI & EDA L & EDA R & PTI \\
\hline $\mathrm{I}$ & 1.637 & 1.239 & 1.947 & 2.332 & 1.545 & 1.547 & 3.807 & 3.668 & 0.433 & 7.884 & 5.063 & 1.011 \\
\hline 1.1 & 1.604 & 1.224 & 1.834 & 2.572 & 1.644 & 1.372 & 3.703 & 4.338 & 0.483 & 7.684 & 4.732 & 1.813 \\
\hline 1.2 & 1.535 & 1.20 & 1.947 & 2.988 & 2.004 & 1.393 & $3.88 I$ & 4.729 & 0.270 & 7.846 & 4.527 & 0.858 \\
\hline 1.3 & 1.435 & 1.180 & 1.449 & 2.948 & 2.208 & 1.725 & 3.973 & 4.722 & 1.144 & 7.955 & 4.574 & 1.438 \\
\hline 1.4 & 1.422 & 1.180 & 1.687 & 2.347 & 1.934 & 1.722 & 4. 170 & 4.692 & 0.735 & 8.829 & 5.415 & 0.721 \\
\hline 1.5 & 1.399 & 1.169 & 1.540 & 2.630 & 2.218 & 1.066 & 4.169 & 4.528 & 0.665 & 7.647 & 5.240 & 1.265 \\
\hline
\end{tabular}

Abbreviations: EDA, electrodermal activity; PTI, pointwise transinformation; L, left; R, right. 
activities calculated from EDA during the resting state is significantly correlated with seizure-like symptoms presented as cognitive, affective, memory, sensory, behavioral, and somatic symptoms in depressive and alcohol-dependent patients, and that a higher level of transinformation measured by PTI, likely reflects temporal lobe epileptiform processes in these patients. ${ }^{25,26}$ The results suggest that decreased PTI during specific cerebellar stimulation found in this study and decreased PTI related to a lower level of seizure-like symptoms $s^{25,26}$ may in principle reflect decreased epileptogenicity, in agreement with findings that cerebral palsy and epilepsy may share some basic pathogenetic mechanisms..$^{5,7,14}$

\section{Conclusion and perspectives}

Further research is warranted, and the results of the present study suggest there are important neurophysiological data that could contribute to explanation of the frequent relationship between cerebral palsy and epilepsy. From a clinical point of view, these findings suggest that transinformation measured by PTI, between left and right autonomic neural activities calculated from EDA reflecting decreased interhemispheric information transfer during cerebellar stimulation may present a physiological indicator that could distinguish an optimal level of stimulation in patients with cerebral palsy. Perspectively, this could provide a clinically useful objective method of increasing treatment outcome in cerebral palsy patients treated by stereotactic cerebellar stimulation. Nevertheless, these results need detailed confirmation in further research and reliable clinical evaluation of their usefulness in the therapy of cerebral palsy.

\section{Acknowledgments}

The study was supported by the Charles University grant (PRVOUK), projects provided by Czech Ministry of Education (MSM0021620849, LH11032) and the project "CEITEC - Central European Institute of Technology" (CZ.1.05/1.1.00/02.0068) from European Regional Development Fund.

\section{Disclosure}

The authors report no conflicts of interest in this work.

\section{References}

1. Zamunér AR, Cunha AB, da Silva E, Negri AP, Tudella E, Moreno MA. The influence of motor impairment on autonomic heart rate modulation among children with cerebral palsy. Res Dev Disabil. 2011;32(1): 217-221.

2. Yang TF, Chan RC, Kao CL, et al. Power spectrum analysis of heart rate variability for cerebral palsy patients. Am J Phys Med Rehabil. 2002;81(5):350-354.
3. Korpelainen JT, Sotaniemi KA, Myllylä VV. Asymmetric sweating in stroke: a prospective quantitative study of patients with hemispheral brain infarction. Neurology. 1993;43(6):1211-1214.

4. Linden D, Berlit P. Sympathetic skin responses (SSRs) in monofocal brain lesions: topographical aspects of central sympathetic pathways. Acta Neurol Scand. 1995;91(5):372-376.

5. Jaseja H. Treatment of interictal epileptiform discharges in cerebral palsy patients without clinical epilepsy: hope for a better outcome in prognosis. Clin Neurol Neurosurg. 2007;109(3):221-224.

6. Jaseja H. Vagal nerve stimulation: exploring its efficacy and success for an improved prognosis and quality of life in cerebral palsy patients. Clin Neurol Neurosurg. 2008;110(8):755-762.

7. Davis R. Cerebellar stimulation for cerebral palsy spasticity, function, and seizures. Arch Med Res. 2000;31(3):290-299.

8. Galanda M, Horvath S. Stereotactic stimulation of the anterior lobe of the cerebellum in cerebral palsy from a suboccipital approach. Acta Neurochir Suppl. 2007;97(Pt 2):239-243.

9. Galanda M, Horvath S. Effect of stereotactic high-frequency stimulation in the anterior lobe of the cerebellum in cerebral palsy: a new suboccipital approach. Stereotact Funct Neurosurg. 2003;80(1-4): 102-107.

10. Penn RD. Chronic cerebellar stimulation for cerebral palsy: a review. Neurosurgery. 1982;10(1):116-121.

11. Cooper IS, Amin I, Upton A, Riklan M, Watkins S, McLellan L. Safety and efficacy of chronic stimulation. Neurosurgery. 1977;1(2): 203-205.

12. Cooper IS, Riklan M, Amin I, Waltz JM, Cullinan T. Chronic cerebellar stimulation in cerebral palsy. Neurology. 1976;26(8):744-753.

13. Riklan M, Cullinan T, Cooper IS. Tension reduction and alerting in man following chronic cerebellar stimulation for the relief of spasticity or intractable seizures. J Nerv Ment Dis. 1977;164(3): 176-181.

14. Sellier E, Uldall P, Calado E, et al. Epilepsy and cerebral palsy: characteristics and trends in children born in1976-1998. Eur J Paediatr Neurol. 2012;16(1):48-55.

15. Machado AG, Shoji A, Ballester G, Marino R Jr. Mapping of the rat's motor area after hemispherectomy: The hemispheres as potentially independent motor brains. Epilepsia. 2003;44(4):500-506.

16. Critchley HD. Electrodermal responses: what happens in the brain. Neuroscientist. 2002;8(2):132-142.

17. Mangina CA, Beuzeron-Mangina JH. Direct electrical stimulation of specific human brain structures and bilateral electrodermal activity. Int J Psychophysiol. 1996;22(1-2):1-8.

18. Phelps EA, O'Connor KJ, Gatenby JC, Gore JC, Grillon C, Davis M. Activation of the left amygdala to a cognitive representation of fear. Nat Neurosci. 2001;4(4):437-441.

19. Bouscein W. Electrodermal Activity. New York: Plenum; 1992.

20. Kantz H, Schreiber T. Nonlinear Time Series Analysis. Cambridge: Cambridge University Press; 1997.

21. Lambertz M, Grebe R, Langhorst P. Phase transitions in the common brainstem and related systems investigated by nonstationary time series analysis. J Auton Nerv Syst. 2000;78(2-3):141-157.

22. Liang H, Ding M, Bressler SL. Temporal dynamics of information flow in the cerebral cortex. Neurocomputing. 2001;38-40:1429-1435.

23. Weinand ME, Labiner DM, Ahern GL. Temporal lobe seizure interhemispheric propagation time depends on nonepileptic cortical cerebral blood flow. Epilepsy Res. 2001;44(1):33-39.

24. Weinand ME, Hussain N, Labiner DM, Ahern GL. Correlation of electrocorticographic to clinical seizure onset and interhemispheric propagation times in temporal lobe epilepsy. Pathophysiology. 2006;13(4): 233-236.

25. Bob P, Jasova D, Raboch J. Subclinical epileptiform process in patients with unipolar depression and its indirect psychophysiological manifestations. PLoS One. 2011;6(11):e28041.

26. Bob P, Jasova D, Bizik G, Raboch J. Epileptiform activity in alcohol dependent patients and possibilities of its indirect measurement. PLoS One. 2011;6(4):e18678. 


\section{Publish your work in this journal}

Neuropsychiatric Disease and Treatment is an international, peerreviewed journal of clinical therapeutics and pharmacology focusing on concise rapid reporting of clinical or pre-clinical studies on a range of neuropsychiatric and neurological disorders. This journal is indexed on PubMed Central, the 'PsycINFO' database and CAS.

The manuscript management system is completely online and includes a very quick and fair peer-review system, which is all easy to use. Visit http://www.dovepress.com/testimonials.php to read real quotes from published authors.

\footnotetext{
Submit your manuscript here: http://www.dovepress.com/neuropsychiatric-disease-and-treatment-journal
} 